\title{
MHD Williamson Nanofluid Flow over a Stretching Sheet through a Porous Medium under Effects of Joule Heating, Nonlinear Thermal Radiation, Heat Generation/Absorption, and Chemical Reaction
}

\author{
J. Bouslimi ${ }^{\mathbb{D}},{ }^{1}$ M. Omri, ${ }^{2}$ R. A. Mohamed, ${ }^{3}$ K. H. Mahmoud, ${ }^{4}$ S. M. Abo-Dahab $\mathbb{i},{ }^{3,5}$ \\ and M. S. Soliman ${ }^{3}$ \\ ${ }^{1}$ Department of Physics, College of Science, Taif University, P. O. Box 11099, Taif 21944, Saudi Arabia \\ ${ }^{2}$ Deanship of Scientific Research, King Abdulaziz University, Jeddah, Saudi Arabia \\ ${ }^{3}$ Mathematics Department, Faculty of Science, South Valley University, Qena 83523, Egypt \\ ${ }^{4}$ Department of Physics, College of Khurma University College, Taif University, P.O. Box 11099, Taif 21944, Saudi Arabia \\ ${ }^{5}$ Computer Science Department, Faculty of Computers and Information, Luxor University, Luxor, Egypt
}

Correspondence should be addressed to J. Bouslimi; jamelabouiyes4@gmail.com

Received 1 April 2021; Accepted 25 May 2021; Published 21 July 2021

Academic Editor: Mustafa Inc

Copyright ( 2021 J. Bouslimi et al. This is an open access article distributed under the Creative Commons Attribution License, which permits unrestricted use, distribution, and reproduction in any medium, provided the original work is properly cited.

\begin{abstract}
In this article, the effect of electromagnetic force with the effect of thermal radiation on the Williamson nanofluid on a stretching surface through a porous medium was studied considering the effect of both heat generation/absorption and Joule heating. On the other hand, the effect of Brownian motion and thermophoresis coefficients was considered. The system of nonlinear partial differential equations governing the study of fluid flow has transformed into a system of ordinary differential equations using similarity transformations and nondimensional variables which were subsequently solved numerically by using the Rung-Kutta fourth-order method with shooting technique. Moreover, the effect of the resulting physical parameters on the distributions of velocity, temperature, and concentration of nanoparticles has been studied by using graphical forms with an interest in providing physical meanings to each parameter. Finally, special diagrams were made to explain the study of the effect of some physical parameters on the skin friction coefficient and the local Nusselt number; these results led to reinforcement in the values of the skin friction coefficient for the increased values of the magnetic field and the Darcy number while the effect on the local Nusselt number by thermal radiation as well as the heat generation/absorption coefficients became negative.
\end{abstract}

\section{Introduction}

In recent years, the study of non-Newtonian fluids has received the attention of researchers in the field of hydrodynamics around the world due to the enormous scientific developments in their applications. The Williamson fluid is one of the most important non-Newtonian fluids characterized by less viscosity with an increase in the rate of shear stress and very similar in its properties of polymeric solutions, for example. In another meaning, in the Williamson fluid model, the effective viscosity should lessen indefinitely with the rising shear rate, which is nothing but infinite viscosity at stationary and nil viscosity as the shear rate tends to infinity. A model of Williamson has been discovered by Williamson [1] in 1929, while Subbarayudu et al. [2] investigated the assessment of time-dependent flow of Williamson fluid with radiative blood flow against a wedge. On the other hand, Lyubimova et al. [3] analyzed the stability of quasiequilibrium states and supercritical regimes of thermal vibrational convection of Williamson fluid in zero gravity conditions, but multiple solutions for MHD transient flow of Williamson nanofluids with convective heat transport 
were studied by Hashim et al. [4]. Hamid et al. [5] discussed an investigation of thermal and solutal stratification effects on mixed convection flow and heat transfer of Williamson nanofluid.

Nanofluids are a modern class of fluids discovered by Choi [6]. Researchers in the field of studying fluids around the world have paid attention to this type of fluids, which plays an important role in many modern technological applications that serve life. On the other hand, nanofluids enter many industries, for example, the satellite industry. Nanofluids also have an important role in the medical fields, for example, also in the use of nanoparticles of gold in the treatment of cancerous tumors, as well as the manufacture of microscopic bombs that are used to eliminate cancerous tumors as well. Sajadifar et al. [7] examined fluid flow and heat transfer of non-Newtonian nanofluid in a microtube considering slip velocity and temperature jump boundary conditions, while Khan and Pop [8] investigated boundarylayer flow of a nanofluid past a stretching sheet, and at the same time, Farooq et al. [9] analyzed MHD stagnation point flow of viscoelastic nanofluid with nonlinear radiation effects. Very recently, Ahmed et al. [10] explained magnetohydrodynamic Maxwell nanofluid flow over a stretching surface through a porous medium: effects of nonlinear thermal radiation, convective boundary conditions, and heat generation/absorption. Alshomrani [11] studied generalized Fourier's and Fick's laws in bioconvection flow of magnetized Burgers nanofluid utilizing motile microorganisms. Alshomrani [12] investigated numerical investigation for bioconvection flow of viscoelastic nanofluid with magnetic dipole and motile microorganisms. Loganathan and Rajan [13] excogited an entropy approach of Williamson nanofluid flow with Joule heating and zero nanoparticle mass flux. It was found from this study that the velocity distribution decreases with increasing the values of the magnetic field parameter while the temperature distribution increases with increasing the values of this parameter. Khan et al. [14] illustrated evaluating the characteristics of the magnetic dipole for shearthinning Williamson nanofluid with thermal radiation. Khan et al. [15] represented changes in viscosity of Williamson nanofluid flow due to thermal and solutal stratification. Hayat et al. [16] explained mixed convective three-dimensional flow of Williamson nanofluid subject to chemical reaction. Ramzan et al. [17] analyzed Darcy-Forchheimer 3D Williamson nanofluid flow with generalized Fourier and Fick's laws in a stratified medium. It was observed from this study that the velocity distribution was decreased by the influence of the magnetic field parameter and also by the influence of the Williamson fluid parameter.

Electromagnetic fluid dynamics (magnetohydrodynamic fluid) is the study of fluids that have the characteristic of electromagnetic conductivity such as plasma fluids, metal fluids, and salt water, and the first to start studying this field is Alfvén [18] in 1942. Mohamed et al. [19] studied MHD Jeffrey nanofluid flow over a stretching sheet through a porous medium in the presence of nonlinear thermal radiation and heat generation/absorption. Also, Chandrashekar et al. [20] examined a discontinuous Galerkin method for a twodimensional reduced resistive MHD model. A numerical study of MHD mixed convection under volumetric heat source in a vertical square duct with wall effects investigated by Liu et al. [21]. Tassone et al. [22] excogitated MHD mixed convection flow in the WCLL: heat transfer analysis and cooling system optimization. Motsa et al. [23] illuminated the spectral relaxation method and spectral quasilinearization. Khan et al. [24] studied peristaltic transport of a Jeffrey fluid with variable viscosity through a porous medium in an asymmetric channel. Ellahi [25] elucidated a study on the convergence of series solutions of non-Newtonian thirdgrade fluid with variable viscosity by means of the homotopy analysis method. Umar et al. [26] investigated numerical treatment for the three-dimensional Eyring-Powell fluid flow over a stretching sheet with velocity slip and activation energy. Ramesh et al. [27] explained heat transfer in MHD dusty boundary layer flow over an inclined stretching sheet with nonuniform heat source/sink.

Joule heating (also referred to as resistive or ohmic heating) describes the process where the energy of an electric current is converted into heat as it flows through a resistance. The explanation for this is that when electrical current flows through solid or liquid materials that conduct electricity, electrical energy is converted into heat energy through the occurrence of resistances inside the conductor. In this case, free electrons transfer energy by collisions. Ramzan et al. [28] studied radiative and Joule heating effects in the MHD flow of a micropolar fluid with partial slip and convective boundary condition. Mohamed et al. [29] discussed the thermal radiation and MHD effects on free convective flow of a polar fluid through a porous medium in the presence of internal heat generation and chemical reaction. Also, Tetbirt et al. [30] discussed a numerical study of magnetic effect on the velocity distribution field in a macro-/microscale of a micropolar and viscous fluid in a vertical channel. At some time, Ghadikolaei et al. [31] investigated numerical study on magnetohydrodynamic CNT-water nanofluids as a micropolar dusty fluid influenced by nonlinear thermal radiation and joule heating effect. It is worth noting that Gireesha et al. [32] investigated hall effects on dusty nanofluid twophase transient flow past a stretching sheet using the KVL model. It was also reported that Aghanajafi et al. [33] explained numerical simulation of laminar forced convection of water-CuO nanofluid inside a triangular duct. But Hussain et al. [34] examined the effects of viscous dissipation and Joule heating on MHD Sisko nanofluid over a stretching cylinder. Moreover, free convective heat and mass transfer of MHD non-Newtonian nanofluids over a cone in the presence of a nonuniform heat source/sink was studied by Raju et al. [35]. Hayat et al. [36] excogitated radiative flow of Jeffrey fluid in a porous medium with power law heat flux and heat source. Meanwhile, Hartnett and Kostic [37] analyzed heat transfer to Newtonian and nonNewtonian fluids in rectangular ducts.

The main objective of the present work is to study the flow of a non-Newtonian Williamson fluid that contains nanoparticles on a stretching sheet through a porous medium under the influences of the magnetic field, nonlinear thermal radiation, and Joule heating in the presence of heat generation/absorption and chemical reaction on the distributions of 
velocity, temperature, and concentration of nanoparticles taking into account studying effects of the Brownian motion coefficient and thermophoresis coefficient. On the other hand, the similarity transformations and nondimensional variables were used in converting the system of partial differential equations governing the movement of flow into a system of ordinary differential equations, which were solved by using the Runge-Kutta numerical method with a shooting technique. The graphs were used to study the effects of all physical parameters on the distributions of velocity, temperature, and concentration of nanoparticles.

\section{Formulation of the Problem}

In the beginning, the study of Williamson nanofluid flow can be shown in several steps: the first step is the fluid flow being steady and incompressible, the second step is that the flowing process is two-dimensional flow on a stretching surface through a porous medium, and the third step is the plate is stretched along the $x$-axis with a velocity $U_{w}(x)=B x$, where $B$ is the stretching rate. The basic equations for the balance of mass, momentum, energy, and nanoparticle volume fraction of the flow problem can be expressed in vector form as follows.

2.1. Continuity Equation. The continuity equation for conservation of mass becomes

$$
\nabla \cdot \vec{q}=0
$$

where $\nabla$ is the differential operator and $\vec{q}$ is the flow velocity vector.

2.2. Conservation of Momentum Equation. The NavierStokes equation for the balance of linear momentum is given by

$$
\rho_{f}\left[\frac{\partial \vec{q}}{\partial t}+(\vec{q} \cdot \nabla) \vec{q}\right]=\nabla \cdot \bar{S}+\vec{F}_{e}-\frac{\mu}{K} \vec{q},
$$

where $\rho_{f}$ is the density of the nanofluid, $t$ is the time, $\bar{S}$ is the Cauchy stress tensor, $\mu$ is the coefficient of dynamic viscosity, $\vec{F}_{e}=\vec{J} \times \vec{B}$ is the Lorentz force produced by the interaction of the applied magnetic field with velocity of fluid, $\vec{J}=\sigma(\vec{E}$ $+\vec{q} \times \vec{B})$ is the current density, $\vec{B}$ is the external magnetic field, $\sigma$ is the electrical conductivity, and $K$ is the permeability of the porous medium.

2.3. Conservation of Energy Equation. In the absence of viscous dissipation effects, the conservation of energy for heat transfer is given by the constitutive equation as

$$
\begin{aligned}
\left(\rho c_{P}\right)_{f} & {\left[\frac{\partial T}{\partial t}+(\vec{q} \cdot \nabla) T\right] } \\
= & \nabla \cdot(k \nabla T)+\left(\rho c_{P}\right)_{p}\left[D_{B} \nabla C \cdot \nabla T+\frac{D_{T}}{T_{\infty}}(\nabla T \cdot \nabla T)\right] \\
& \quad-\nabla q_{r}+J_{h}+Q\left(T-T_{\infty}\right),
\end{aligned}
$$

where $c_{P}$ is the specific heat at constant pressure; $\left(\rho c_{P}\right)_{f}$ and $\left(\rho c_{P}\right)_{p}$ are the specific heat of the nanofluid and the nanoparticles, respectively; $T$ is the temperature of the nanofluid; $k$ is the thermal conductivity; $D_{B}$ is the Brownian diffusion coefficient; $D_{T}$ is the thermophoresis diffusion coefficient; $T_{\infty}$ is the ambient temperature; $q_{r}$ is the radiative heat flux; $J_{h}=$ $\vec{F}_{e} \cdot \vec{q}$ is the Joule heating, and $Q$ is the uniform volumetric heat generation/absorption.

2.4. Conservation of Nanoparticle Concentration Equation. For a homogeneous chemical reaction [38], the concentration equation of nanoparticle volume fraction becomes

$$
\frac{\partial C}{\partial t}+(\vec{q} \cdot \nabla) C=D_{B} \nabla^{2} C+\frac{D_{T}}{T_{\infty}} \nabla^{2} T-R^{*}\left(C-C_{\infty}\right),
$$

where $C$ is the nanoparticle volume fraction and the rates $R^{*}>0$ and $R^{*}<0$ denote destructive and constructive reaction rates, respectively.

For the Williamson fluid model, Cauchy stress tensor $\bar{S}$ is defined as [26]

$$
\begin{aligned}
& \bar{S}=-P \bar{I}+\bar{\tau}, \\
& \bar{\tau}=\left(\mu_{\infty}+\frac{\mu_{0}-\mu_{\infty}}{1-\Gamma \dot{\gamma}}\right) \bar{A}_{1},
\end{aligned}
$$

where $P$ is pressure, $\bar{I}$ is the unit tensor, $\bar{\tau}$ is the extra stress tensor, $\mu_{0}$ is limiting viscosity at zero shear rate, $\mu_{\infty}$ is limiting viscosity at the infinite shear rate, $\Gamma>0$ is a time constant, $\bar{A}_{1}$ is the first Rivlin-Erickson tensor, and $\gamma$ is defined as follows:

$$
\dot{\gamma}=\left(\frac{\pi}{2}\right)^{1 / 2}, \quad \pi=\operatorname{trace}\left(\bar{A}_{1}\right)^{2} .
$$

Here, it is considered the case for $\mu_{\infty}=0$ and $\Gamma \gamma<1$; thus, Equation (6) can be written as follows:

$$
\bar{\tau}=\left[\frac{\mu_{0}}{1-\Gamma \dot{\gamma}}\right] \bar{A}_{1}=\mu_{0}(1+\Gamma \dot{\gamma}) \bar{A}_{1} .
$$

The right-hand side of Equation (8) is done by using binomial expansion. Making use of Equations (5) and (8) into Equations (1) to (4), the two-dimensional boundary layer equations governing the flow can be written as follows [39]:

$$
\frac{\partial u}{\partial x}+\frac{\partial u}{\partial y}=0,
$$




$$
\begin{aligned}
& u \frac{\partial u}{\partial x}+v \frac{\partial u}{\partial y}= v \frac{\partial^{2} u}{\partial y^{2}}+\sqrt{2} v \Gamma \frac{\partial u}{\partial y} \frac{\partial^{2} u}{\partial y^{2}}-\frac{\sigma B_{0}^{2}}{\rho} u-\frac{v}{K} u \\
& u \frac{\partial T}{\partial x}+v \frac{\partial T}{\partial y}= \alpha \frac{\partial^{2} T}{\partial y^{2}}-\frac{1}{\left(\rho c_{P}\right)_{f}} \frac{\partial q_{r}}{\partial y}+\frac{\left(\rho c_{P}\right)_{p}}{\left(\rho c_{P}\right)_{f}} \\
& \cdot\left[D_{B}\left(\frac{\partial C}{\partial y} \frac{\partial T}{\partial y}\right)+\left(\frac{D_{T}}{T_{\infty}}\right)\left(\frac{\partial T}{\partial y}\right)^{2}\right] \\
&+\frac{\sigma B_{o}^{2}}{\left(\rho c_{P}\right)_{f}} u^{2}+\frac{Q}{\left(\rho c_{P}\right)_{f}}\left(T-T_{\infty}\right) \\
& u \frac{\partial C}{\partial x}+v \frac{\partial C}{\partial y}=D_{B} \frac{\partial^{2} C}{\partial y^{2}}+\frac{D_{T}}{T_{\infty}} \frac{\partial^{2} T}{\partial y^{2}}-R^{*}\left(C-C_{\infty}\right)
\end{aligned}
$$

where $u$ is the velocity components in the direction of the $x$ -axis and $v$ is the velocity components in the direction of the $y$-axis; in addition, $\alpha$ represent the thermal diffusivity, $\rho$ refer to density of the fluid, and $v$ is the kinematic viscosity of fluid; also, $T$ and $T_{\infty}$, respectively, are fluid temperature and ambient fluid temperature. A uniform magnetic field of strength $B_{0}$ is applied in the transverse direction of the flow; due to the small magnetic Reynolds number, it is not necessary to introduce the effect of the induced magnetic field. It should be noted that $D_{B}, D_{T}, \sigma, c_{P}$, and $R^{*}$ are, respectively, the Brownian diffusion coefficient, the thermophoresis diffusion coefficient, the electrical conductivity, the specific heat at constant pressure, and destructive and constructive reaction rates. Here, $C$ and $C_{\infty}$ are the concentration of nanoparticles and ambient nanoparticle concentration. It is worth noting that the effect of nonlinear thermal radiation, heat source/sink, and Joule heating is taken in the energy equation. The viscous dissipation is assumed to be negligibly small in the energy equation. The effects of the homogenous chemical reaction are taken in the concentration equation.

The boundary conditions for the present investigation problem can be written in the formula:

$$
u=U_{w}(x), v=0, T=T_{w}, D_{B} \frac{\partial C}{\partial y}+\frac{D_{T}}{T_{\infty}} \frac{\partial T}{\partial y}=0, \quad \text { at } y=0,
$$

$$
u \longrightarrow 0, v \longrightarrow 0, T \longrightarrow T_{\infty}, C \longrightarrow C_{\infty}, \quad \text { as } y \longrightarrow \infty
$$

where $U_{w}(x)=B x$ represent the stretching surface velocity and $B>0$ is the stretching rate, and the radiation heat flux $q_{r}$ can be written as follows:

$$
\begin{aligned}
q_{r} & =-\frac{4 \sigma^{*}}{3 k^{*}} \frac{\partial T^{4}}{\partial y}=-\frac{16 \sigma^{*}}{3 k^{*}} T^{3} \frac{\partial T}{\partial y}, \\
\therefore \frac{1}{\left(\rho c_{P}\right)_{f}} \frac{\partial}{\partial y}\left(q_{r}\right) & =\frac{1}{\left(\rho c_{P}\right)_{f}} \frac{\partial}{\partial y}\left(-\frac{4 \sigma^{*}}{3 k^{*}} \frac{\partial T^{4}}{\partial y}\right) \\
& =-\frac{16 \sigma^{*}}{3 k^{*}\left(\rho c_{P}\right)_{f}} \frac{\partial}{\partial y}\left(T^{3} \frac{\partial T}{\partial y}\right) ;
\end{aligned}
$$

when substituting by Equation (16) into Equation (11), the energy equation takes the following form:

$$
\begin{aligned}
u \frac{\partial T}{\partial x}+v \frac{\partial T}{\partial y}= & \alpha \frac{\partial^{2} T}{\partial y^{2}}+\frac{16 \sigma^{*}}{3 k^{*}\left(\rho c_{P}\right)_{f}} \frac{\partial}{\partial y}\left(T^{3} \frac{\partial T}{\partial y}\right) \\
& +\frac{\left(\rho c_{P}\right)_{p}}{\left(\rho c_{P}\right)_{f}}\left[D_{B}\left(\frac{\partial C}{\partial y} \frac{\partial T}{\partial y}\right)+\left(\frac{D_{T}}{T_{\infty}}\right)\left(\frac{\partial T}{\partial y}\right)^{2}\right] \\
& +\frac{\sigma B_{o}^{2}}{\left(\rho c_{P}\right)_{f}} u^{2}+\frac{Q}{\left(\rho c_{P}\right)_{f}}\left(T-T_{\infty}\right)
\end{aligned}
$$

where $\sigma^{*}$ and $k^{*}$ are the Stefan-Boltzmann constant and mean absorption coefficient; the similarity transformations and nondimensional variables can be written as follows:

$$
\begin{aligned}
\eta & =\sqrt{\frac{U_{w}(x)}{v x}} y, u=B x f^{\prime}(\eta), v=-\sqrt{B v} f(\eta), \theta(\eta) \\
& =\frac{T-T_{\infty}}{T_{w}-T_{\infty}}, \phi(\eta)=\frac{C-C_{\infty}}{C_{\infty}}
\end{aligned}
$$

where $\eta$ is the similarity variable and $f(\eta), \theta(\eta)$, and $\phi(\eta)$, respectively, are the dimensionless stream function, temperature, and concentration of nanoparticles; the similarity transformations and the nondimensional variables (18) were used to convert the boundary layer governing partial differential Equations (10), (12), and (17) to a set of ordinary differential equations taking the mathematical formulas:

$$
\begin{aligned}
& f^{\prime \prime \prime}(\eta)-\left(f^{\prime}(\eta)\right)^{2}+f(\eta) f^{\prime \prime}(\eta) \\
& +\lambda f^{\prime \prime}(\eta) f^{\prime \prime \prime}(\eta)-M f^{\prime}(\eta)-\operatorname{Da} f^{\prime}(\eta)=0, \\
& \theta^{\prime \prime}(\eta)+\operatorname{Pr}\left(R\left(\left(1+\left(\theta_{w}-1\right) \theta(\eta)\right)^{3} \theta^{\prime}(\eta)\right)^{\prime}\right. \\
& +\operatorname{Nb} \theta^{\prime}(\eta) \phi^{\prime}(\eta)+S \theta(\eta)+\operatorname{Nt}\left(\theta^{\prime}(\eta)\right)^{2} \\
& \left.+\operatorname{MEc}\left(f^{\prime}(\eta)\right)^{2}+\theta^{\prime}(\eta) f(\eta)\right)=0, \\
& \phi^{\prime \prime}(\eta)+\operatorname{Le} \phi^{\prime}(\eta) f(\eta)-\operatorname{Le} \gamma \phi(\eta)+\left(\frac{\mathrm{Nt}}{\mathrm{Nb}}\right) \theta^{\prime \prime}(\eta)=0,
\end{aligned}
$$

where the boundary conditions (13) and (14) can be written after converting as new follows:

$$
\begin{gathered}
f(0)=0, f^{\prime}(0)=1, \theta(\eta)=1, \mathrm{Nb} \phi^{\prime}(0)+\mathrm{Nt} \theta^{\prime}(0)=0, \\
f^{\prime}(\infty) \longrightarrow 0, \theta(\infty) \longrightarrow 0, \phi(\infty) \longrightarrow 0,
\end{gathered}
$$

with the knowledge that $M=\sigma B_{0}{ }^{2} / \rho B$ is the magnetic field parameter, $R=16 \sigma^{*} T_{\infty}^{3} / 3 k k^{*}$ is the nonlinear thermal radiation parameter, $\mathrm{Pr}=v / \alpha$ is the Prandtl number, $\mathrm{Nb}=\left(\rho c_{P}\right)_{p}$ $D_{B} C_{\infty} / v\left(\rho c_{P}\right)_{f}$ is the Brownian motion parameter, $\mathrm{Nt}=$ 
TABLE 1: A comparison between the numerical results of Khan and Pop [8] and the results of the current study through the values of $-\theta^{\prime}(0)$ in the case of $\operatorname{Pr}=\mathrm{Le}=10$ and in the absence of both $M, \mathrm{Da}, \mathrm{Ec}, \gamma, S$, and $\lambda$.

\begin{tabular}{|c|c|c|c|c|c|c|}
\hline \multirow[t]{2}{*}{$\mathrm{Nt}$} & \multicolumn{2}{|c|}{$\begin{array}{c}\mathrm{Nb}=0.1 \\
-\theta^{\prime}(0)\end{array}$} & \multicolumn{2}{|c|}{$\begin{array}{c}\mathrm{Nb}=0.2 \\
-\theta^{\prime}(0)\end{array}$} & \multicolumn{2}{|c|}{$\begin{array}{c}\mathrm{Nb}=0.3 \\
-\theta^{\prime}(0)\end{array}$} \\
\hline & Khan and Pop [8] & Present study & Khan and Pop [8] & Present study & Khan and Pop [8] & Present study \\
\hline 0.1 & 0.9524 & 0.9501 & 0.5056 & 0.5065 & 0.2522 & 0.2534 \\
\hline 0.2 & 0.6932 & 0.6910 & 0.3654 & 0.3646 & 0.1816 & 0.1912 \\
\hline 0.3 & 0.5201 & 0.5237 & 0.2731 & 0.2735 & 0.1355 & 0.1382 \\
\hline 0.4 & 0.4026 & 0.4083 & 0.2110 & 0.2124 & 0.1046 & 0.1086 \\
\hline 0.5 & 0.3211 & 0.3270 & 0.1681 & 0.1710 & 0.0833 & 0.0880 \\
\hline
\end{tabular}

$\left(\rho c_{P}\right)_{p} D_{T}\left(T_{w}-T_{\infty}\right) /\left(\rho c_{P}\right)_{f} v T_{\infty}$ is the thermophoresis parameter, $\mathrm{Le}=\nu / D_{B}$ is the Lewis number, $\mathrm{Da}=\mu / \rho B K$ is the Darcy number, $S=Q /\left(\rho c_{P}\right)_{f} B$ is the heat generation $(S>0)$ or absorption $(S<0)$ parameter, $\gamma=R^{*} / B$ is the chemical reaction parameter, $\mathrm{Ec}=U_{w}^{2}(x) / c_{P}\left(T_{w}-T_{\infty}\right)$ is the Eckert number, and finally $\lambda=\Gamma x \sqrt{2 B^{3} / v}$ is the non-Newtonian Williamson parameter.

Expression for the local Nusselt number $\mathrm{Nu}_{x}$ and the skin friction coefficient $C_{f}$ are defined as

$$
\begin{aligned}
\mathrm{Nu}_{x} & =\frac{x q_{w}}{k}\left(T_{w}-T_{\infty}\right), \\
C_{f} & =\frac{\tau_{w}}{\rho U_{w}^{2}(x)} .
\end{aligned}
$$

And the reader will note that the dimensionless mass flux represented by a Sherwood number $\mathrm{Sh}_{x}$ is now identically zero and $q_{w}$ and $\tau_{w}$ are the heat flux and the shear stress along the stretching surface, respectively, on the following mathematical formulas:

$$
\begin{aligned}
& q_{w}=-\alpha\left(\frac{\partial T}{\partial y}\right)_{y=0}+\left(q_{r}\right)_{y=0}, \\
& \tau_{w}=\mu\left[\frac{\partial u}{\partial y}+\frac{\Gamma}{\sqrt{2}}\left(\frac{\partial u}{\partial y}\right)^{2}\right]_{y=0} .
\end{aligned}
$$

When substituting Equations (26) and (27) into Equations (24) and (25), the mathematical nondimensional formulas of Equations (24) and (25) are

$$
\begin{gathered}
\frac{\mathrm{Nu}_{x}}{\sqrt{\mathrm{Re}_{x}}}=-\left(1+R \theta_{w}{ }^{3}\right) \theta^{\prime}(0), \\
\sqrt{\mathrm{Re}_{x}} C_{f}=f^{\prime \prime}(0)\left(1+\frac{\lambda}{2} f^{\prime \prime}(0)\right),
\end{gathered}
$$

where $\operatorname{Re}_{x}=x U_{w}(x) / \nu$ is the local Reynolds number based on the stretching velocity $U_{w}(x)$. It should be noted that in the absence of a magnetic field, porous medium, nonlinear thermal radiation, heat generation/absorption, and finally the chemical reaction, the work is due to Nadeem and Hussain [39].

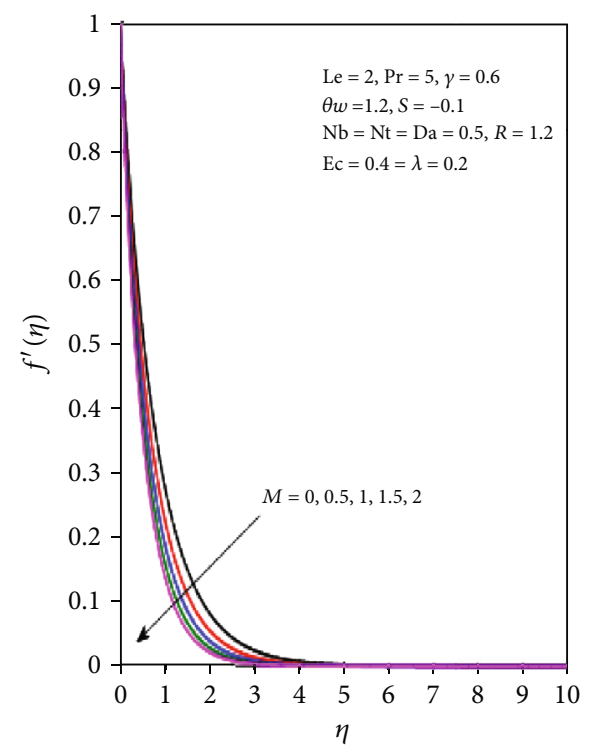

Figure 1: The velocity profile for different values of $M$.

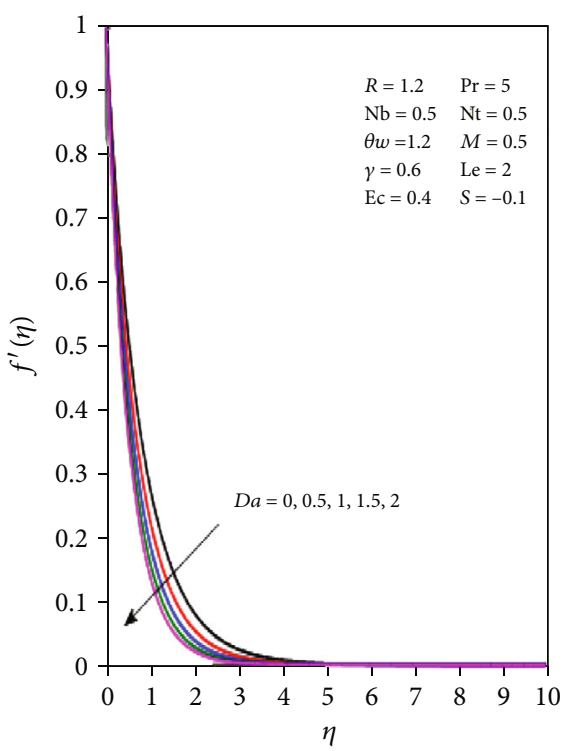

FIgURE 2: The velocity profile for different values of Da. 


\section{Numerical Solution}

The Runge-Kutta numerical method is one of the most important numerical analysis methods that are used in solving a system of ordinary differential equations through which the differential equation is reduced degree to make it easier and simpler to solve. On the other hand, there are different formulas for the solution by the numerical method of Runge-Kutta method; for example, there is the RungeKutta method of the fourth order and also the RungeKutta method of the fifth order, and the most used method is the Runge-Kutta method of the fourth order because it gives accurate results and is easy to use, and its derivation depends on the Euler method. The numerical solutions of the current study are listed in several steps. In the first step, the system of partial differential Equations (10), (12), and
(17) for the current study has been converted into a system of ordinary differential Equations (19)-(21) using the similarity transformation and nondimensional variables (18); also, the boundary conditions (13) and (14) have been converted into the new forms (22) and (23). In the second step, the appropriate numerical method used in solving the new system of prior equations is the Runge-Kutta fourth-order with shooting technique, and by the way, this method was used to reduce the order of differential equations. In the third step, the program used to solve the system of ordinary differential equations is MATLAB, and the step size $\Delta \eta=$ 0.001 is used to obtain the numerical solution with $\eta_{\max }=$ 10; also, it used the bvp4c function in solving these equations. Differential Equations (19)-(21) can be rewritten in the simplest form as follows:

$$
\begin{array}{r}
f^{\prime \prime \prime}(\eta)=\frac{\left(\left(f^{\prime}(\eta)\right)^{2}-f(\eta) f^{\prime \prime}(\eta)+M f^{\prime}(\eta)+\operatorname{Da} f^{\prime}(\eta)\right)}{\left(1+\lambda f^{\prime \prime}(\eta)\right)}, \\
\theta^{\prime \prime}(\eta)=-\frac{\operatorname{Pr}\left(S \theta(\eta)+f(\eta) \theta^{\prime}(\eta)+3 R\left(\theta_{w}-1\right)\left(1+\left(\theta_{w}-1\right) \theta(\eta)\right)^{2}\left(\theta^{\prime}(\eta)\right)^{2}+\operatorname{Nb} \theta^{\prime}(\eta) \phi^{\prime}(\eta)+\operatorname{Nt}\left(\theta^{\prime}(\eta)\right)^{2}+M E c\left(f^{\prime}(\eta)\right)^{2}\right)}{\left(1+\operatorname{Pr} R\left(1+\left(\theta_{w}-1\right) \theta(\eta)\right)^{3}\right)} \\
\phi^{\prime \prime}(\eta)=-\operatorname{Le}^{\prime}(\eta) f(\eta)+\operatorname{Le} \gamma \phi(\eta)-\left(\frac{\mathrm{Nt}}{\mathrm{Nb}}\right) \theta^{\prime \prime}(\eta) .
\end{array}
$$

In the last step, Equations (30)-(32) have been written inside the MATLAB program on the following formulas:

$$
\begin{gathered}
F(1)=\xi(2), \\
F(2)=\xi(3), \\
F(3)=\frac{\left(\xi(2)^{2}-\xi(1) \xi(3)+M \xi(2)+\mathrm{Da} \xi(2)\right)}{1+\lambda \xi(3)}, \\
F(4)=\xi(5), \\
F(5)=-\frac{\operatorname{Pr}\left(S \xi(4)+\xi(1) \xi(5)+\operatorname{Nb} \xi(5) \xi(7)+\operatorname{Nt}(\xi(5))^{2}+M \operatorname{Ec}(\xi(2))^{2}+3 R(\xi(5))^{2}\left(\theta_{w}-1\right)\left(1+\left(\theta_{w}-1\right) \xi(4)\right)^{2}\right)}{\left(1+\operatorname{Pr} R\left(1+\left(\theta_{w}-1\right) \xi(4)\right)^{3}\right)} \\
F(6)=\xi(7), \\
F(7)=-\operatorname{Le} \xi(7) \xi(1)+\operatorname{Le} \gamma \xi(6)-\left(\frac{\mathrm{Nt}}{\mathrm{Nb}}\right) \xi(5) .
\end{gathered}
$$


With the boundary conditions:

$$
\begin{gathered}
\xi_{a}(1)=0, \xi_{a}(2)=1, \xi_{a}(4)=1, \mathrm{Nb} \xi_{a}(7)+\mathrm{Nt} \xi_{a}(5)=0, \\
\xi_{b}(2)=0, \xi_{b}(4)=0, \xi_{b}(6)=0 .
\end{gathered}
$$

Taking into account both:

$$
\begin{aligned}
f(\eta) & =\xi(1), f^{\prime}(\eta)=\xi(2), f^{\prime \prime}(\eta)=\xi(3), f^{\prime \prime \prime}(\eta) \\
& =F(3), \theta(\eta)=\xi(4), \theta^{\prime}(\eta)=\xi(5) \\
\theta^{\prime \prime}(\eta) & =F(5), \phi(\eta)=\xi(6), \phi^{\prime}(\eta)=\xi(7), \phi^{\prime \prime}(\eta) \\
& =F(7), f(0)=\xi_{a}(1), f^{\prime}(0)=\xi_{a}(1) \\
\theta(0) & =\xi_{a}(4), \theta^{\prime}(0)=\xi_{a}(5), \phi^{\prime}(0)=\xi_{a}(7), f^{\prime}(\infty) \\
& =\xi_{b}(2), \theta(\infty)=\xi_{b}(4), \phi(\infty)=\xi_{b}(6) .
\end{aligned}
$$

To ensure the accuracy and correctness of the numerical solutions of the current study, a numerical comparison was made between the numerical values and results of the current study with the numerical values and results of the work published by Khan and Pop [8] in Table 1. The great convergence between the two studies was noted, which gives high credibility to the current study.

\section{Results and Discussion}

After converting the system of partial differential equations ruling to study the flow of fluid into a system of ordinary differential equations, it has a set of important parameters that we list in the following order $M, \mathrm{Da}$, and $\lambda$ which are the magnetic field, the Darcy number, and non-Newtonian Williamson parameters, respectively, while $R, \theta_{w}$, and $S$ represent the nonlinear thermal radiation, the ratio temperature, and the heat generation/absorption parameters, respectively; also, Pr is the Prandtl number and Nt is the thermophoresis parameter, while $\mathrm{Ec}, \gamma, \mathrm{Le}$, and $\mathrm{Nb}$ are the Eckert number, the chemical reaction, the Lewis number, and Brownian motion parameters, respectively. The effect of all the previous physical parameters on the velocity, temperature, and concentration of nanoparticle distributions has been studied by making graphical figures that clarify this and by showing the physical meanings of each parameter and its importance in this study, and we can list the results of this study in detail.

4.1. Velocity Distributions. It is known that the rate at which the fluid flows onto a specific surface is entirely determined by the distribution of velocity; therefore, velocity distributions play an important role in studying the changes that occur in the flow rate of the fluid. On the other hand, when affecting the flowing fluid with external forces, the behavior of the fluid's movement is changing, and one of these types of external forces is the magnetic field. Figure 1 shows the effect of the strength of the magnetic field parameter $M$ on the fluid velocity distribution $f^{\prime}(\eta)$. It is noticeable that the result of this effect is negative in the sense that the relationship between the magnetic field and the velocity distribution

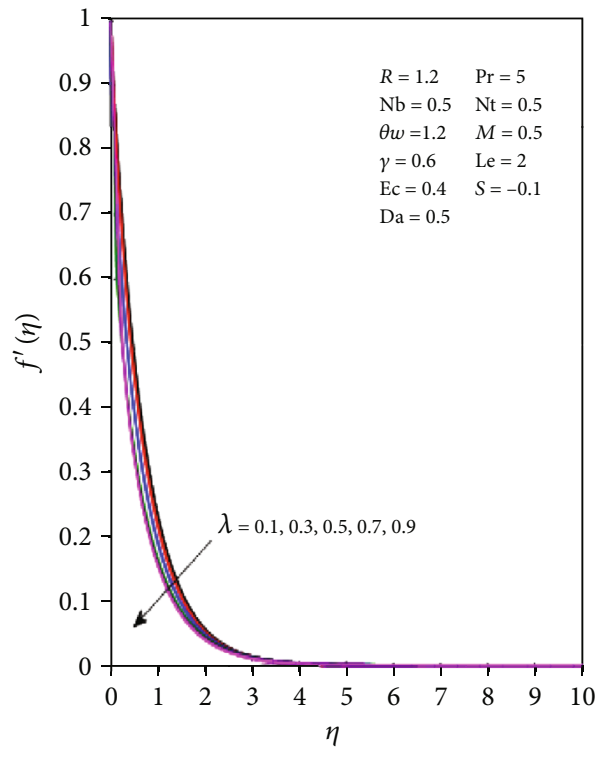

Figure 3: The velocity profile for different values of $\lambda$.

is inverse; the increase in the values of the magnetic field means a decrease in the velocity of the fluid. Physically, when influencing a moving fluid by the magnetic field, the fluid particles are stimulated, which creates a kind of counter force that slows and reduces the fluid's motion; moreover, this force is perpendicular to the velocity vector on the one hand and also perpendicular to the magnetic field vector on the other hand which is originally a resistance force called Lorentz force, while highlighting that the increase in the magnetic field reduces the thickness of the boundary layer. If you want to know the effect of the Darcy number Da on the velocity distribution, you should look at Figure 2. It becomes clear to you that the velocity distribution decreases under the influence of the large values that the Darcy number Da takes. Physically, the porous medium is the medium that contains a group of small voids called pores that are interrupted by the fluid when it moves on this medium. On the other hand, the permeability of the fluid through the porous medium is related to its porosity.

Also, the greater the values of the Darcy number $\mathrm{Da}$, the greater the resistance of the porous medium to the movement of the fluid on it on the one hand, in addition to the fluid viscosity on the other hand, which leads to a decrease in the fluid velocity. Moving to Figure 3, it reviews the effect of the non-Newtonian Williamson coefficient $\lambda$ on the velocity distribution, and it clears that this effect is negative in the sense that the increase in the values of this coefficient is followed by a decrease in velocity of the fluid, which makes its movement slow.

4.2. Temperature Distributions. The change in the fluid temperature plays an important role in the fluid's behavior in addition to its effect also on the particles inside the fluid. Now, we will study the effect of some physical parameters on the fluid temperature distribution $\theta(\eta)$ and note the changes taking place; for example, Figures 4 and 5 illustrate the effects of the nonlinear thermal radiation parameter $R$ 


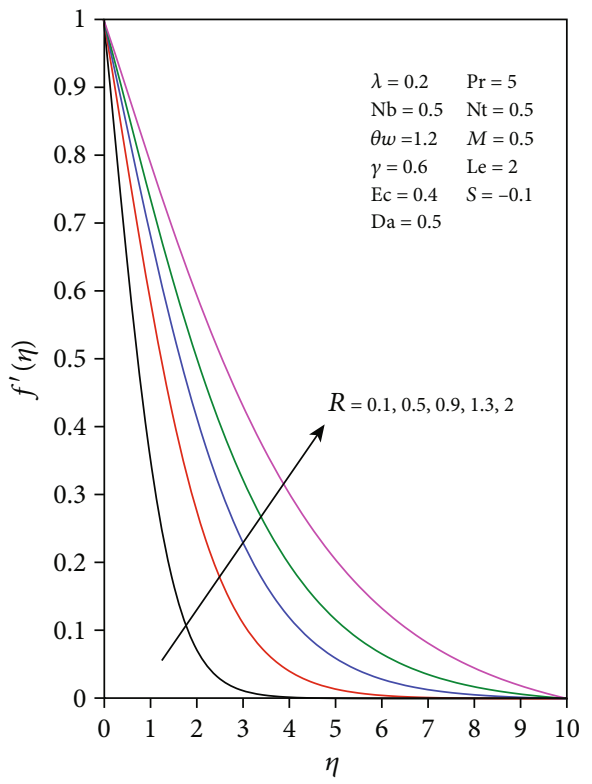

Figure 4: Temperature profile for different values of $R$.

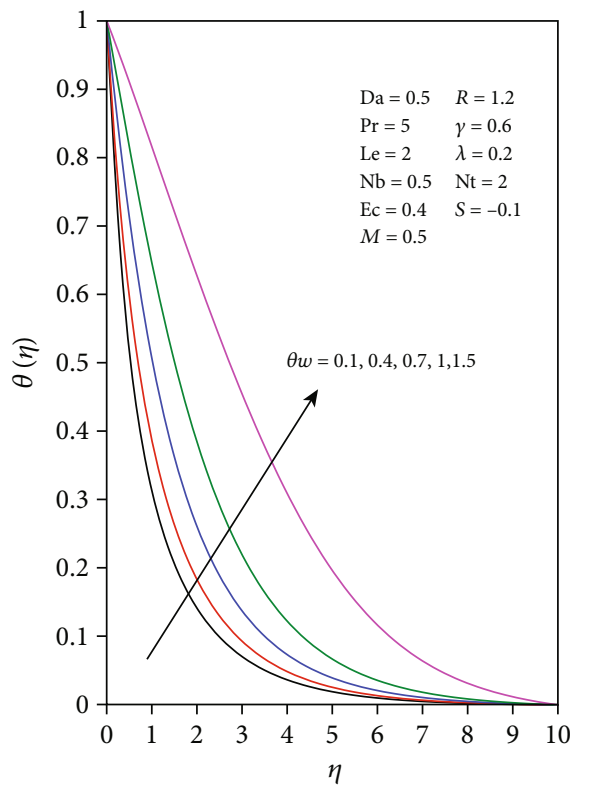

Figure 5: Temperature profile for different values of $\theta_{w}$.

and the ratio temperature parameter $\theta_{w}$ on the temperature distribution $\theta(\eta)$; it was noticed that the large values taken by the nonlinear thermal radiation parameter $R$ and ratio temperature parameter $\theta_{w}$ work to enhance the heat transfer inside the fluid and work to stimulate it. Physically, the positive effect of the nonlinear thermal radiation parameter $R$ on the temperature of the nanofluid leads to three things. Firstly, the heat transfer of all distances of the boundary layer is supported, or in other words, it raises the temperature of the boundary layer regularly. Secondly, it makes the nanoparticles inside the fluid gain thermal energy, which improves the transfer and thermal diffusion within the fluid due to the thermal conductivity of the nanoparticles.

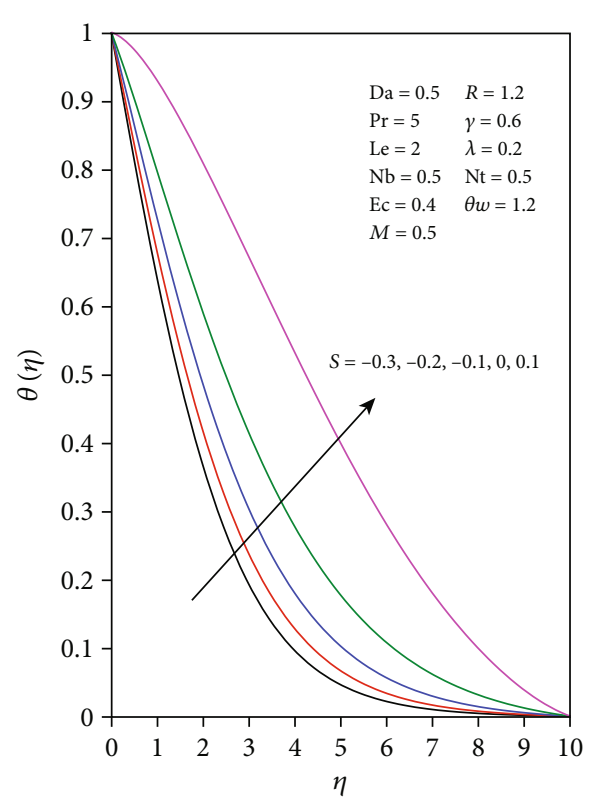

Figure 6: Temperature profile for different values of $S$.

Thirdly, it works to enhance the thermal transfer methods of the nanofluid, which is the thermal transfer method by conduction and the method of thermal transfer by load. On the other hand, the effect of the ratio temperature increases the boundary layer temperature and increases its thickness. When talking about the effect of the heat generation/absorption parameter $S$ on the fluid temperature distribution $\theta(\eta)$, as shown in Figure 6, there is a significant enhancement in the distribution of the fluid temperature due to the enhancement in the values of parameter $S$.

Physically, in the case of the phenomenon of heat generation, there is an enhancement in the transfer and thermal spread of the fluids, which raises temperature of fluid and also raises the temperature and thickness of the boundary layer with the increase in the values of the heat source type $(S>0)$, while the exact opposite occurs in the case of the heat absorption type $(S<0)$, but when moving upward from the state of heat absorption type to the state of heat generation type, there is an improvement in the rate of thermal diffusion and the thickness of the boundary layer. Figure 7 displays the impact of Prandtl number Pr on the temperature distribution $\theta(\eta)$; when this parameter takes a large value, the fluid temperature distribution is decreased. Physically, Prandtl number $\mathrm{Pr}$ is defined as the ratio of momentum diffusivity to thermal diffusivity; on the other hand, there are two types of Prandtl number Pr: the first type is $\operatorname{Pr}>1$; this means that the rate of momentum diffusion dominates the rate of thermal diffusion, and the second type is $\operatorname{Pr}<1$ which urges the opposite, and Figure 7 shows the case in which $\operatorname{Pr}>1$ such that the thermal diffusion within the fluid becomes small; thus, the fluid temperature and thickness of the boundary layer become decreasing. The distribution of the fluid temperature $\theta(\eta)$ changes with the effect of the thermophoresis parameter Nt values; as shown in Figure 8, the direct relationship between them refers to the enhancement in the values of the thermophoresis parameter $\mathrm{Nt}$ leading to an 


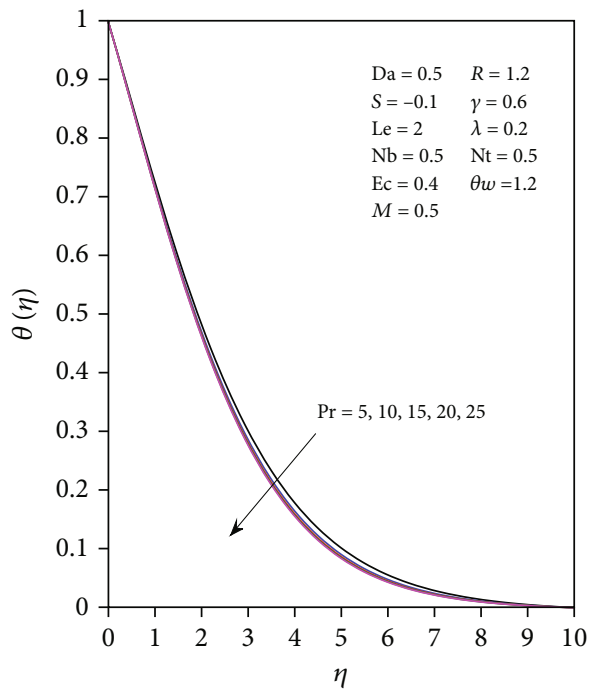

Figure 7: Temperature profile for different values of Pr.

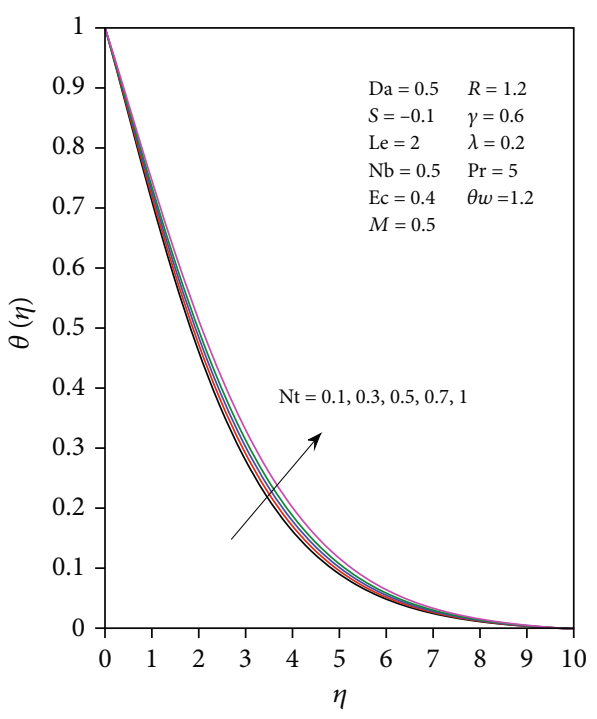

Figure 8: Temperature profile for different values of Nt.

increase in the fluid temperature distribution $\theta(\eta)$. Physically, there are two different sources: the first is hot and the other is cold; the particles close to the hot medium absorb heat energy, which makes them move from the hot medium to the cold medium and raises its temperature by means of convection; this process is called the process of thermal potential difference; this is exactly what happens inside the fluid when it is exposed to a heat source. The fluid particles absorb heat energy that makes it spread throughout the fluid, raising its temperature as well as enhancing the thickness of the boundary layer of the fluid. Figure 9 studies the effect of the Eckert number Ec on the temperature distribution $\theta(\eta)$, so it was noted that the large values that take by this parameter affect the temperature distribution making this effect positive in the sense that it enhances the effect of temperature. Finally, the influence of the coefficient of the magnetic field $M$ as well as the effect of the Darcy number Da on the

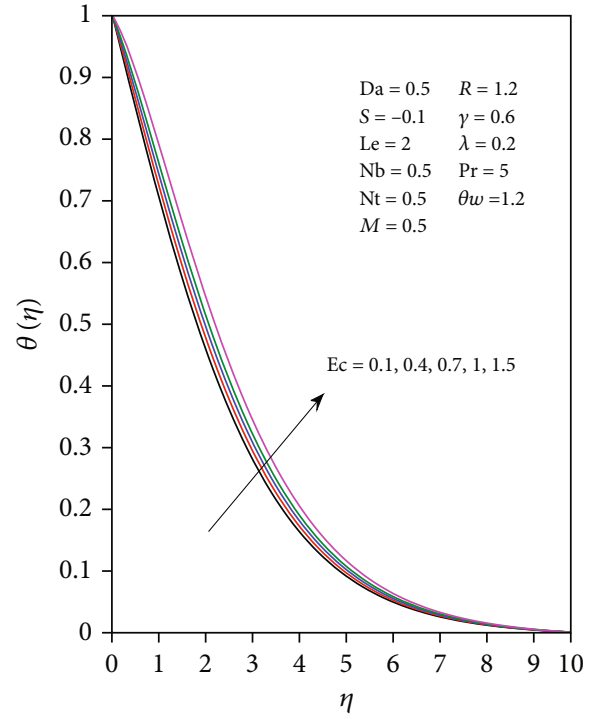

Figure 9: Temperature profile for different values of Ec.

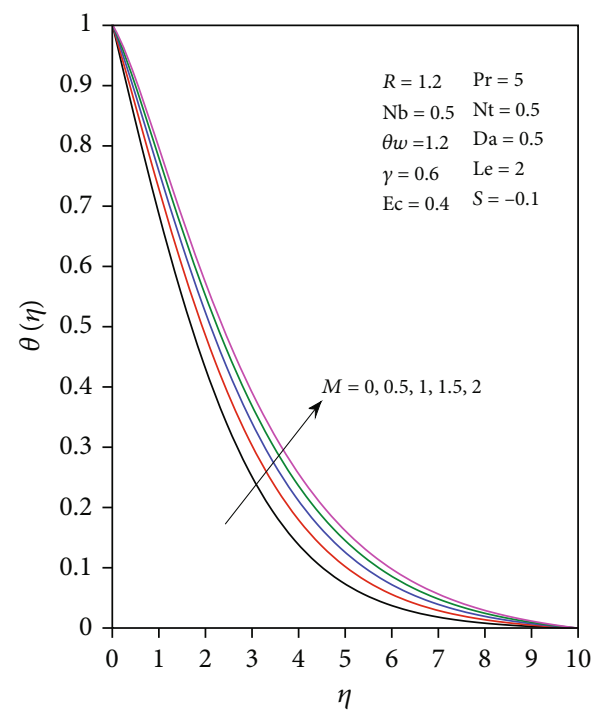

Figure 10: Temperature profile for different values of $M$.

temperature distribution $\theta(\eta)$ is shown graphically in Figures 10 and 11, respectively; it is very clear from the study of the two figures that the fluid temperature and the boundary layer thickness increase with increasing values of these two parameters.

4.3. Concentration of Nanoparticle Distributions. The study of the concentration of nanoparticles within a fluid plays an important role in the properties and applications of this fluid from several sides; for example, the degree and efficiency of the thermal conductivity of a nanofluid are related to the concentration of nanoparticles inside it, and also, the degree and efficiency of its electrical conductivity are related to the concentration of nanoparticles. Figures 12 and 13 display the effect of both the magnetic field parameter $M$ and the Darcy number $\mathrm{Da}$ on the distribution of the concentration of nanoparticles $\phi(\eta)$, and it is clear from the two figures that 


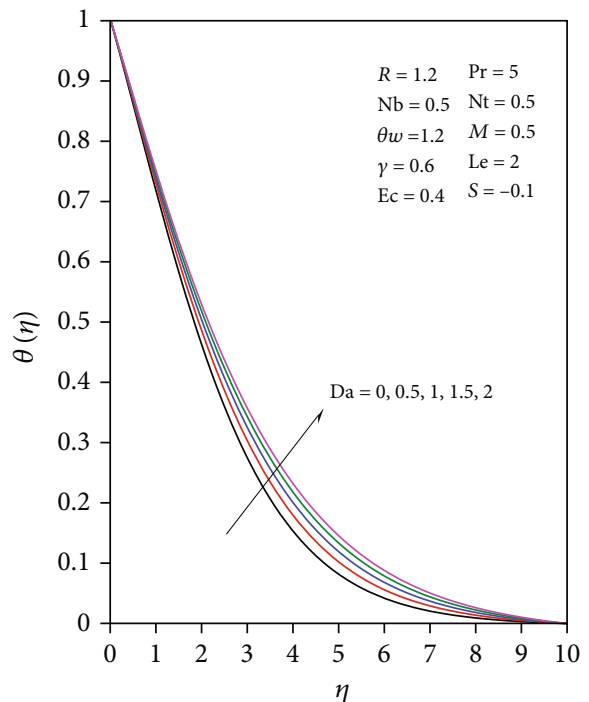

Figure 11: Temperature profile for different values of Da.

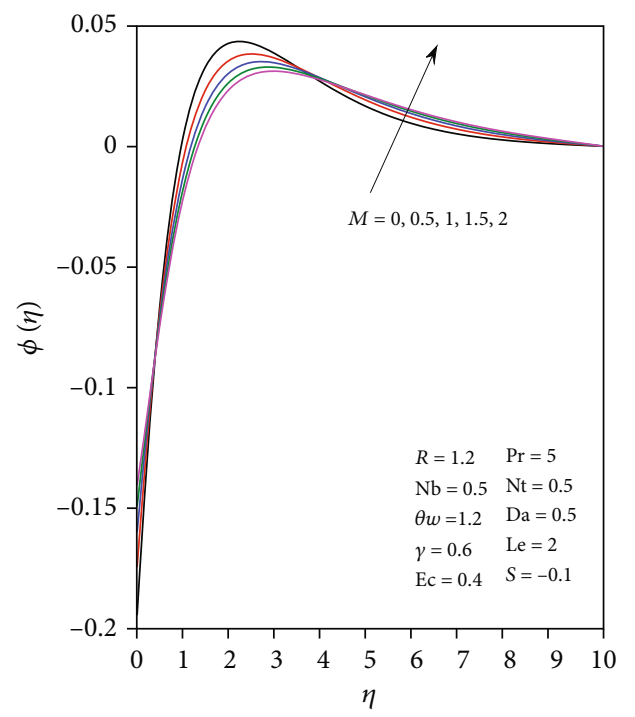

FIGURE 12: Concentration of nanoparticle profile for different values of Pr.

the effect is very positive. Physically, firstly, the effect of the magnetic field $M$ on the fluid works to reduce the movement of the fluid accompanied slowly in the movement of the nanofluid particles, which makes its concentration large. Secondly, the effect of the Darcy number Da on the fluid movement gives a great opportunity for collision of the nanoparticles with the pores of the porous medium, which causes it to accumulate inside the pores and the porous medium itself, and accordingly, the concentration of nanoparticles becomes increasing. On the other hand, Figures 14 and 15 show the effects of the nonlinear thermal radiation parameter $R$ and the ratio temperature parameter $\theta_{w}$ on the concentration of nanoparticle distribution $\phi(\eta)$ within the fluid. It was noted that the reinforcement in the values of these two parameters works to strengthen the temperature of the nanoparticles and thickness of the boundary layer of the fluid, as it

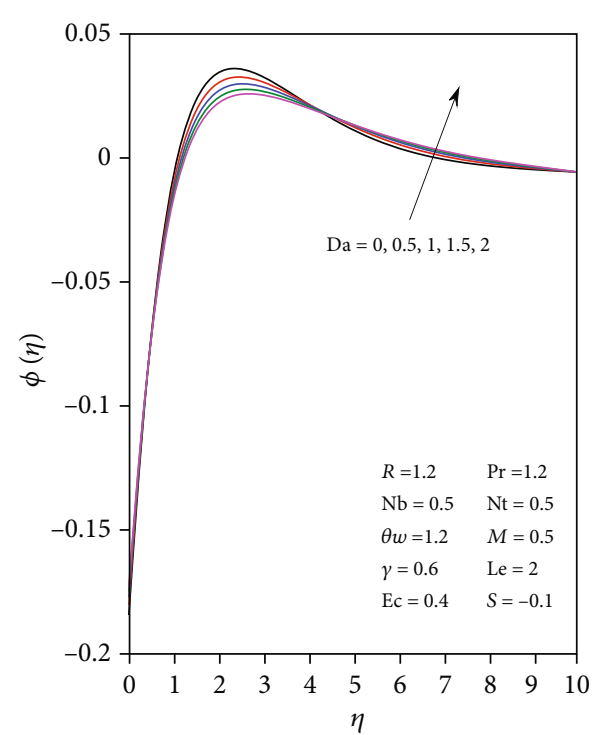

FIGURE 13: Concentration of nanoparticle profile for different values of $\mathrm{Da}$.

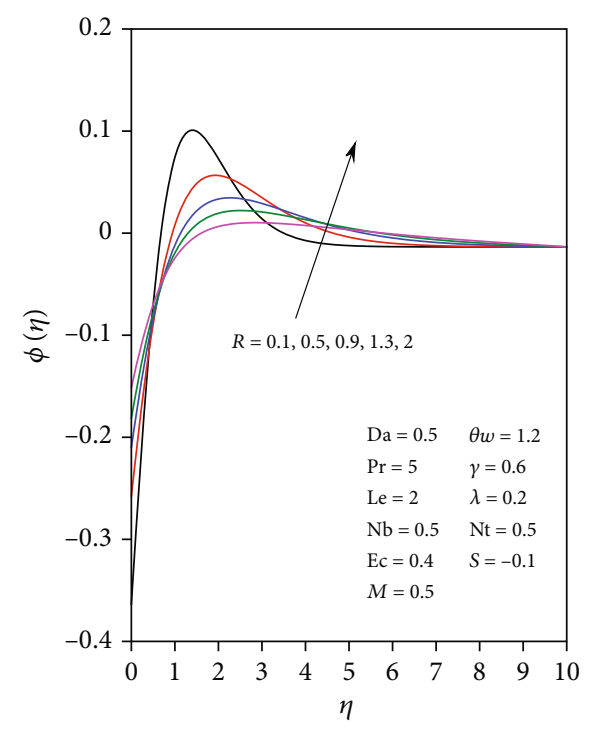

FIGURE 14: Concentration of nanoparticle profile for different values of $R$.

works on a significant increase in the spread of the temperature in all parts of the fluid. Physically, nanoparticle fluid molecules are greatly affected by the radiation source that generates heat, which causes fluid particles to interact positively with this heat, which increases the mechanical energy of the nanoparticles, and this actually leads to their stimulation and increased concentration. The concentration of nanoparticles gives a good or positive impression in the event that it is affected by the heat generation/absorption parameter $S$ because the mechanism of action of this parameter is very similar to the mechanism of nonlinear thermal radiation parameter $R$ only in the case of heat generation; on the other side, the heat generation works to stimulate the temperature of nanoparticles and thus increases the concentration of nanoparticles $\phi(\eta)$ and adds to the positive effect on the 


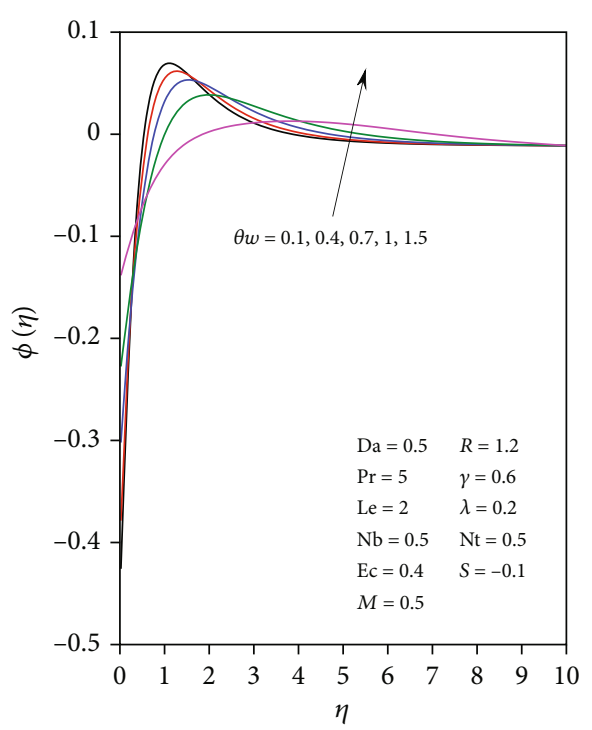

FIGURE 15: Concentration of nanoparticle profile for different values of $\theta_{w}$.

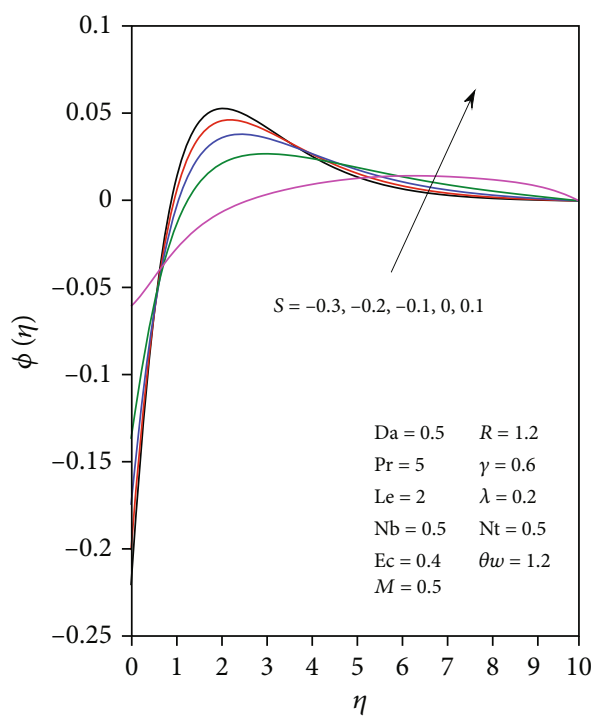

FIGURE 16: Concentration of nanoparticle profile for different values of $S$.

boundary layer in terms of thickness, while in the case of heat absorption, the opposite occurs, and this is shown in Figure 16. As for Figure 17, it shows the negative effect of Prandtl number $\operatorname{Pr}$ on the concentration of nanoparticle distribution $\phi(\eta)$ because in the case where $\operatorname{Pr}>1$, thermal diffusion equipment is very small compared to the momentum diffusion rate, and accordingly, the concentration of nanoparticles becomes small that is the physical meaning. Figures 18 and 19 show the effects of both the Eckert number Ec and the thermophoresis parameter $\mathrm{Nt}$ on the distribution of the concentration of nanoparticles $\phi(\eta)$ within the fluid; it was found that the increase in this parameters gives a large concentration of the nanofluid particles, at a time when the effects of both Lewis number Le and chemical reaction

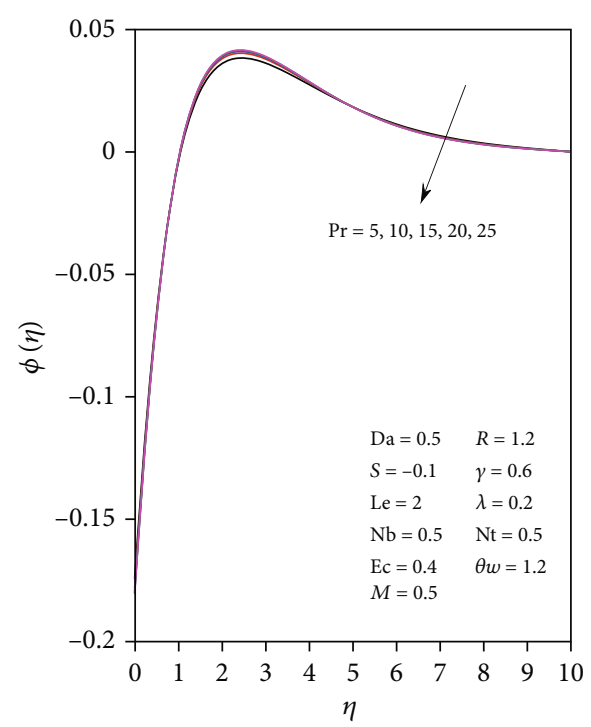

FIGURE 17: Concentration of nanoparticle profile for different values of Pr.

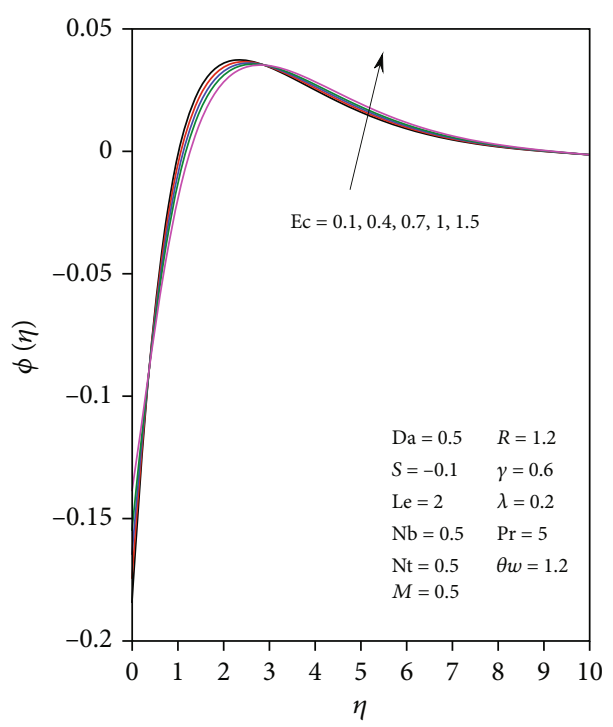

FIGURE 18: Concentration of nanoparticle profile for different values of Ec.

parameter $\gamma$ on the concentration of nanoparticles $\phi(\eta)$ inside the fluid were negative; this is illustrated in Figures 20 and 21. Finally, Figure 22 clears the effect of the Brownian motion parameter $\mathrm{Nb}$ on the distribution of the concentration of nanoparticles $\phi(\eta)$ within the fluid. It is noticeable that the distribution of the concentration of nanoparticles $\phi(\eta)$ decreases in the event that this parameter is enhanced. Physically, the Brownian motion of the nanofluid is a random motion of the nanoparticles present in this fluid in the absence of any external effects so that the movement of these particles is free in all directions, but in the case of a large thermal diffusion resulting from the effect of thermal radiation and the coefficient of thermophoresis, it becomes that the random movement of the nanoparticles is limited. 


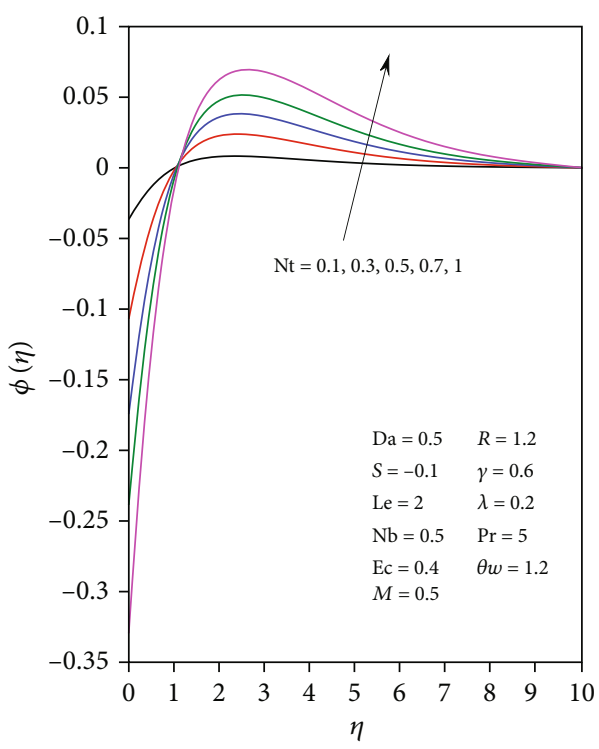

FIGURE 19: Concentration of nanoparticle profile for different values of Nt.

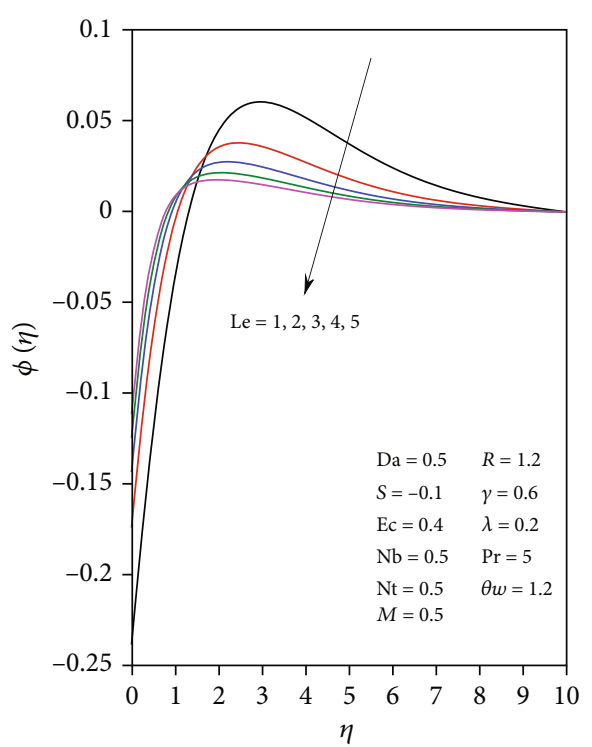

FIGURE 20: Concentration of nanoparticle profile for different values of $S$.

4.4. Skin Friction and Local Nusselt Number Profiles. Figure 23 shows the change in the coefficient of skin friction when affecting it by the coefficient of the magnetic field $M$ and the Darcy number Da. It is clear that the coefficient of skin friction decreases with increasing values of these parameters, meaning that the increase in both the values of the magnetic field and the Darcy number increases the rate of fluid velocity gradient regularly. On the other hand, Figures 24 and 25 show the effects of nonlinear thermal radiation parameter $R$, heat generation/absorption parameter $S$, and ratio temperature parameter $\theta_{w}$ on the local Nusselt number $\mathrm{Nu}_{x} / \sqrt{\mathrm{Re}_{x}}$; it is clear that this effect is negative in the sense of an increase in the values of previous parameters leading to a decrease in the local Nusselt number, and that

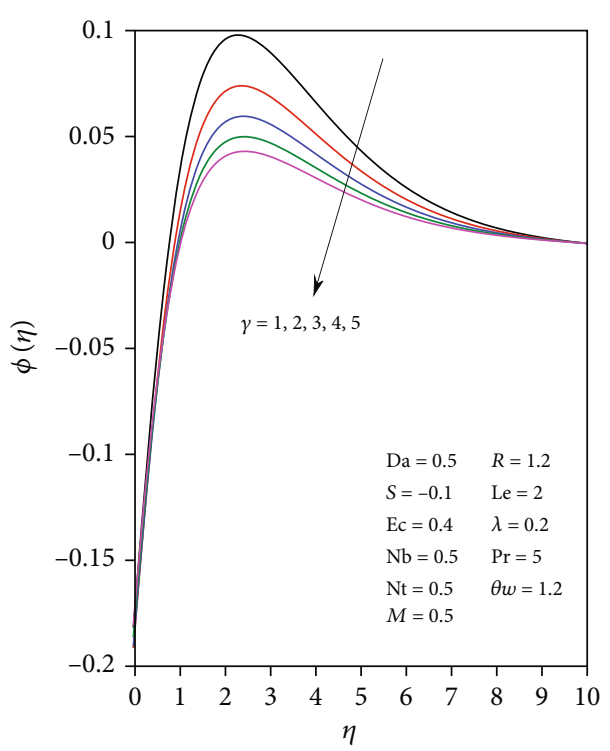

FIgURE 21: Concentration of nanoparticle profile for different values of $\gamma$.

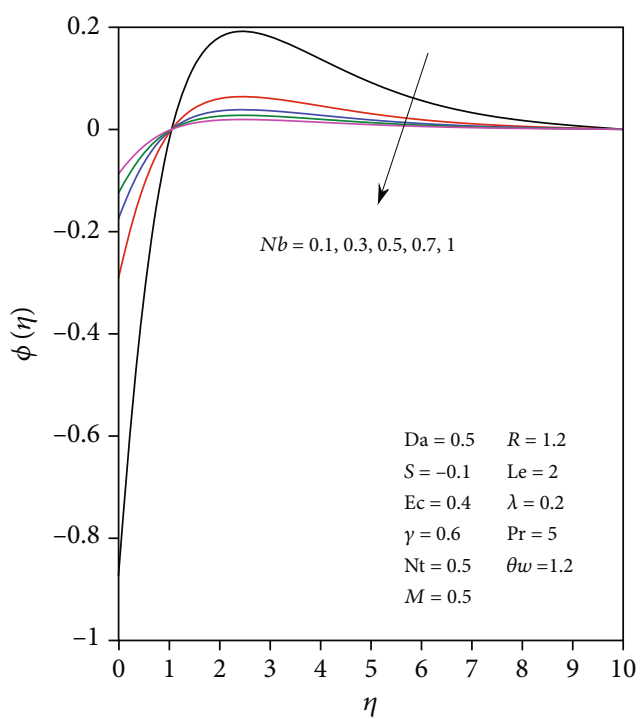

FIGURE 22: Concentration of nanoparticle profile for different values of $\mathrm{Nb}$.

means that the ratio of convective to conductive heat transfer at a boundary in a fluid becomes small. Tables 2 and 3 show the numerical values of the local Nusselt number $\mathrm{Nu}_{x} / \sqrt{\mathrm{Re}_{x}}$ and skin friction coefficient $\sqrt{\operatorname{Re}_{x}} C_{f}$ for all physical parameters resulting from the current numerical study of the Williamson fluid flow process that contains nanoparticles on an expanding surface in the presence of a porous medium. The following was observed that the skin friction coefficient $\sqrt{\mathrm{Re}_{x}} C_{f}$ has become a constant value for all physical parameters with the exception of each of the magnetic field parameter $M$, Darcy number $\mathrm{Da}$, and non-Newtonian Williamson parameter $\lambda$, whereas the values of the local Nusselt number $\mathrm{Nu}_{x} / \sqrt{\mathrm{Re}_{x}}$ decrease under the influence of all physical parameters. 


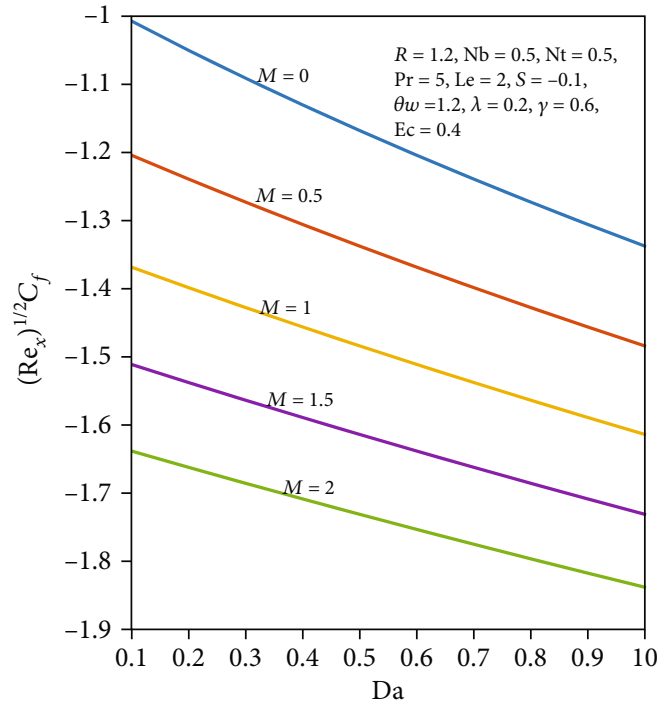

FIgURE 23: Skin friction profile for different values of $M$, Da.

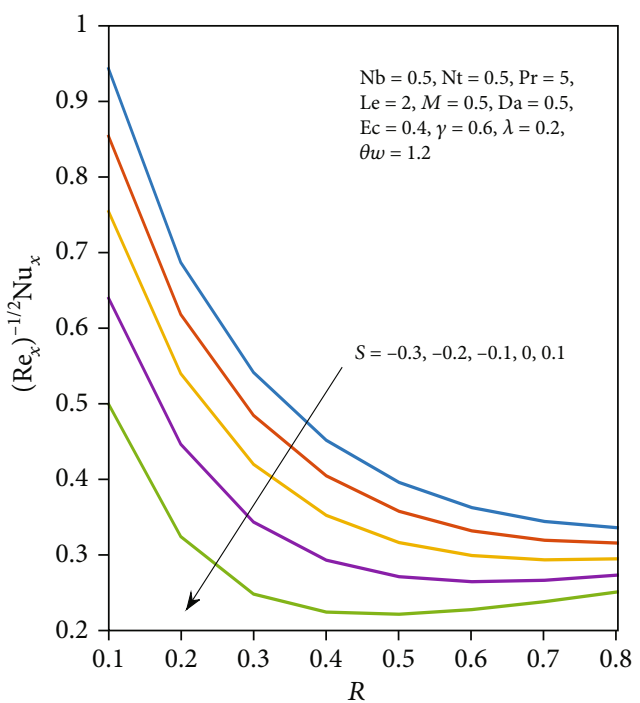

FIGURE 24: Local Nusselt number profile for different values of $S, R$.

\section{Conclusion}

In this article, the two-dimensional electromagnetic flow of the Williamson nanofluid has been studied on a stretching sheet through a porous medium; on the other hand, the governing partial differential equations were converted into a system of ordinary differential equations using the similarity transformations and the nondimensional variable under the effects of nonlinear thermal radiation, heat generation/absorption, chemical reaction, Brownian motion parameter, thermophoresis parameter, magnetic field, Darcy number, Joule heating, Lewis number, Prandtl number, and Williamson non-Newtonian parameter on distribution of velocity, temperature, and concentration of nanoparticles; the most important points drawn from this study are the following:

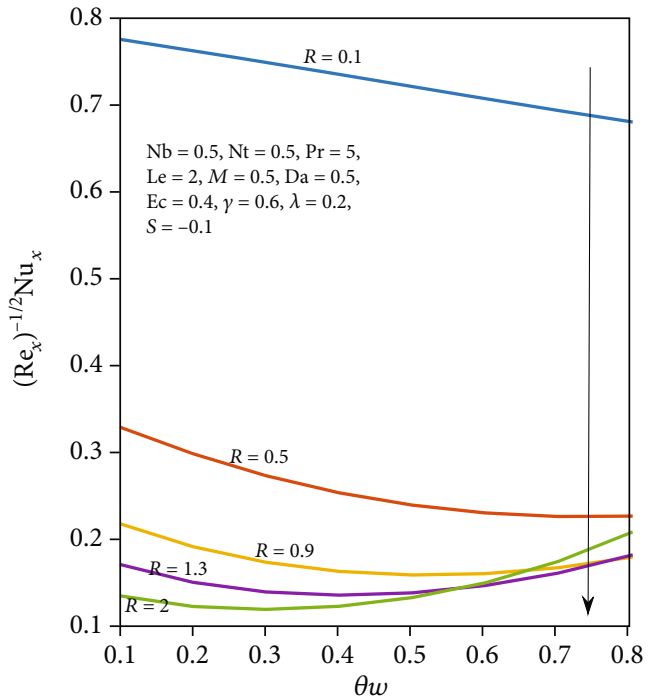

FIgURE 25: Local Nusselt number profile for different values of $S, R$.

TABLE 2: The numerical values of the skin friction coefficient $\sqrt{\mathrm{Re}_{x}} C_{f}$ and the local Nusselt number $\mathrm{Nu}_{x} / \sqrt{\mathrm{Re}_{x}}$ for values of $M$, $\mathrm{Da}$, and $\lambda$ parameters when $R=1.2, \mathrm{Nb}=0.5, \mathrm{Nt}=0.5, \mathrm{Pr}=5$, Le $=2, S=-0.1, \mathrm{Ec}=0.4, \theta_{w}=1.2$, and $\gamma=0.6$.

\begin{tabular}{lcccc}
\hline$M$ & $\mathrm{Da}$ & $\lambda$ & $\sqrt{\mathrm{Re}_{x}} C_{f}$ & $\frac{\mathrm{Nu}_{x}}{\sqrt{\mathrm{Re}_{x}}}$ \\
\hline 0 & 0.5 & 0.2 & -1.16630705 & 0.32673441 \\
0.5 & & & -1.33621158 & 0.32351790 \\
1 & & & -1.48295534 & 0.32112657 \\
1.5 & & & -1.61309297 & 0.31923008 \\
2 & & & -1.73049604 & 0.31766292 \\
0.5 & 0 & 0.2 & -1.16630705 & 0.32463205 \\
& 0.5 & & -1.33621158 & 0.32351790 \\
& 1 & & -1.48295534 & 0.32272607 \\
& 1.5 & & -1.61309297 & 0.32212446 \\
& 2 & & -1.73049604 & 0.32164656 \\
0.5 & 0.5 & 0.1 & -1.37751997 & 0.32368673 \\
& & 0.3 & -1.28773536 & 0.32332326 \\
& & 0.5 & 17.61631263 & 0.32263272 \\
& & 0.7 & 0.36239912 & 0.32190223 \\
\hline
\end{tabular}

(i) The velocity distribution $f^{\prime}(\eta)$ of the fluid is negatively affected by the influence of the magnetic field parameter $M$, the Darcy number $\mathrm{Da}$, and the nonNewtonian Williamson parameter $\lambda$ at the time when their influence on the distributions of the temperature $\theta(\eta)$ and concentration of the nanoparticles $\phi(\eta)$ becomes positive

(ii) The enhancement in the values of nonlinear thermal radiation $R$ parameter and the ratio temperature $\theta_{w}$ resulted in the enhancement of both temperature $\theta$ $(\eta)$ of the fluid and the concentration of the nanoparticles $\phi(\eta)$, while the opposite occurs when increasing the values of Prandtl number Pr 
TABLE 3: The numerical values of the skin friction coefficient $\sqrt{\operatorname{Re}_{x}} C_{f}$ and the local Nusselt number $\mathrm{Nu}_{x} / \sqrt{\operatorname{Re}_{x}}$ for all values of physical parameter when $M=\mathrm{Da}=0.5$ and $\lambda=0.2$.

\begin{tabular}{|c|c|c|c|c|c|c|c|c|c|c|}
\hline$R$ & $\mathrm{Nb}$ & $\mathrm{Nt}$ & $\operatorname{Pr}$ & Le & $S$ & Ec & $\theta_{w}$ & $\gamma$ & $\sqrt{\operatorname{Re}_{x}} C_{f}$ & $\frac{\mathrm{Nu}_{x}}{\sqrt{\mathrm{Re}_{x}}}$ \\
\hline 0.1 & 0.5 & 0.5 & 5 & 2 & -0.1 & 0.4 & 1.2 & 0.6 & -1.33621230 & 0.64166769 \\
\hline 0.5 & & & & & & & & & -1.33621158 & 0.27220459 \\
\hline 0.9 & & & & & & & & & -1.33621158 & 0.28419960 \\
\hline \multirow[t]{3}{*}{1.2} & 0.1 & 0.5 & 5 & 2 & -0.1 & 0.4 & 1.2 & 0.6 & -1.33621158 & 0.32351790 \\
\hline & 0.3 & & & & & & & & -1.33621158 & 0.32351790 \\
\hline & 0.5 & & & & & & & & -1.33621158 & 0.32351790 \\
\hline \multirow[t]{3}{*}{1.2} & 0.5 & 0.1 & 5 & 2 & -0.1 & 0.4 & 1.2 & 0.6 & -1.33621158 & 0.32410873 \\
\hline & & 0.3 & & & & & & & -1.33621158 & 0.32381315 \\
\hline & & 0.5 & & & & & & & -1.33621158 & 0.32351790 \\
\hline \multirow[t]{3}{*}{1.2} & 0.5 & 0.5 & 5 & 2 & -0.1 & 0.4 & 1.2 & 0.6 & -1.33621158 & 0.32351790 \\
\hline & & & 10 & & & & & & -1.33621158 & 0.31230129 \\
\hline & & & 15 & & & & & & -1.33621158 & 0.30971540 \\
\hline \multirow[t]{3}{*}{1.2} & 0.5 & 0.5 & 5 & 1 & -0.1 & 0.4 & 1.2 & 0.6 & -1.33621158 & 0.32356291 \\
\hline & & & & 2 & & & & & -1.33621158 & 0.32351790 \\
\hline & & & & 3 & & & & & -1.33621158 & 0.32349773 \\
\hline \multirow[t]{3}{*}{1.2} & 0.5 & 0.5 & 5 & 2 & -0.3 & 0.4 & 1.2 & 0.6 & -1.33621158 & 0.34389846 \\
\hline & & & & & -0.2 & & & & -1.33621158 & 0.33376466 \\
\hline & & & & & -0.1 & & & & -1.33621158 & 0.32351790 \\
\hline \multirow[t]{3}{*}{1.2} & 0.5 & 0.5 & 5 & 2 & -0.1 & 0.1 & 1.2 & 0.6 & -1.33621158 & 0.32487121 \\
\hline & & & & & & 0.4 & & & -1.33621158 & 0.32351790 \\
\hline & & & & & & 0.7 & & & -1.33621158 & 0.32216467 \\
\hline \multirow[t]{3}{*}{1.2} & 0.5 & 0.5 & 5 & 2 & -0.1 & 0.4 & 0.1 & 0.6 & -1.33621158 & 0.18104819 \\
\hline & & & & & & & 0.4 & & -1.33621158 & 0.14127077 \\
\hline & & & & & & & 0.7 & & -1.33621158 & 0.16205303 \\
\hline \multirow[t]{3}{*}{1.2} & 0.5 & 0.5 & 5 & 2 & -0.1 & 0.4 & 1.2 & 1 & -1.33621158 & 0.32350105 \\
\hline & & & & & & & & 2 & -1.33621158 & 0.32347976 \\
\hline & & & & & & & & 3 & -1.33621158 & 0.32346885 \\
\hline
\end{tabular}

(iii) It was found that the distribution of the concentration of nanoparticles $\phi(\eta)$ decreased under the influences of the Lewis number Le, Brownian motion coefficient $\mathrm{Nb}$, and chemical reaction parameter $\gamma$

(iv) The increase and enhancement in the effects of both heat generation/absorption $S$ parameter and the Eckert number Ec resulted in an increase and enhancement in the distributions of both temperature $\theta(\eta)$ and concentration of nanoparticles $\phi(\eta)$

(v) Effects of heat generation/absorption parameter $S$, the nonlinear thermal radiation parameter $R$, and the ratio temperature parameter $\theta_{w}$ have a negative effect on the local Nusselt number $\mathrm{Nu}_{x} / \sqrt{\mathrm{Re}_{x}}$ so that the effects of the magnetic field $M$ and Darcy number $\mathrm{Da}$ on the skin friction coefficient $\sqrt{\mathrm{Re}_{x}} C_{f}$ were negative

\section{Symbols}

$\bar{A}_{1}: \quad$ First Rivlin-Erickson tensor
$\vec{B}: \quad$ External magnetic field

$B_{o}: \quad$ Uniform magnetic field

$B$ : $\quad$ Stretching parameter

$C$ : Nanoparticle volume fraction

$C_{w}: \quad$ Nanoparticle fraction at wall

$c_{P}: \quad$ Specific heat at constant pressure

$C_{f}$ : $\quad$ Skin friction coefficient

$D_{B}$ : Brownian diffusion coefficient

$D_{T}$ : Thermophoresis diffusion coefficient

Da: Darcy number

$\vec{E}$. $\quad$ Electric field intensity

Ec: $\quad$ Eckert number

$\vec{F}_{e}: \quad$ The Lorentz force

I: $\quad$ Unit vector

$\vec{J}$ : $\quad$ The current density

$J_{h}: \quad$ The Joule heating

$K$ : $\quad$ Permeability

$k$ : Thermal conductivity

$k^{*}$ : Mean absorption coefficient

Le: Lewis number 
M: $\quad$ Magnetic field parameter

$\mathrm{Nb}$ : Brownian motion parameter

Nt: Thermophoresis parameter

$\mathrm{Nu}_{x}: \quad$ Nusselt number

$P: \quad$ Pressure

Q: Uniform volumetric heat source/sink

$\vec{q}$ : Velocity vector

$q_{w}: \quad$ Heat flux

$q_{m}: \quad$ Mass flux

$q_{r}$ : Nonlinear radiative heat flux

$R: \quad$ Thermal radiation parameter

$R^{*}: \quad$ Constructive reaction rates

$\mathrm{Re}_{x}$ : Local Reynolds number

$S: \quad$ Heat source/sink parameter

$\bar{S}$ : $\quad$ Cauchy stress tensor

$\mathrm{Sh}_{x}$ : Local Sherwood number

$T: \quad$ Fluid temperature

$T_{w}$ : $\quad$ Fluid temperature at wall

$T_{\infty}$ : Ambient fluid temperature

$t: \quad$ Time

$U_{w}: \quad$ Fluid velocity

$u, v: \quad$ Velocity components

$x, y: \quad$ Space coordinates

$\alpha$ : Thermal diffusivity

$v$ : $\quad$ Kinematic viscosity

$\rho: \quad$ Density of the fluid

$\bar{\tau}: \quad$ Extra stress tensor

$\tau_{w}: \quad$ Shear stress along stretching surface

$\sigma^{*}: \quad$ Stefan-Boltzmann constant

$\lambda$ : Non-Newtonian Williamson parameter

$\eta: \quad$ Similarity variable

$\psi: \quad$ Dimensionless stream function

$\phi$ : Dimensionless concentration function

$\theta$ : Dimensionless temperature

$\Gamma: \quad$ Time constant

$\theta_{w}: \quad$ Ratio temperature

$\left(\rho c_{P}\right)_{p}$ : Heat capacity of a nanoparticle

$\left(\rho c_{P}\right)_{f}$ : Heat capacity of the base fluid

$\mu_{0}$ : Limiting viscosity at zero shear rate

$\mu_{\infty}$ : Limiting viscosity at infinite

$\gamma$ : Chemical reaction parameter.

\section{Data Availability}

No data were used to support the findings of this study.

\section{Conflicts of Interest}

The authors have no conflicts of interest regarding the publication of the paper.

\section{Acknowledgments}

The authors would like to acknowledge the financial support of Taif University researchers, supporting project no. TURSP-2020/162, Taif University, Saudi Arabia.

\section{References}

[1] R. V. Williamson, "The flow of pseudoplastic materials," Industrial and Engineering Chemistry, vol. 21, no. 11, pp. 1108-1111, 1929.

[2] K. Subbarayudu, S. Suneetha, and P. B. A. Reddy, “The assessment of time dependent flow of Williamson fluid with radiative blood flow against a wedge," Propulsion and Power Research, vol. 9, no. 1, pp. 87-99, 2020.

[3] T. P. Lyubimova, A. V. Perminov, and M. G. Kazimardanov, "Stability of quasi-equilibrium states and supercritical regimes of thermal vibrational convection of a Williamson fluid in zero gravity conditions," International Journal of Heat and Mass Transfer, vol. 129, pp. 406-414, 2019.

[4] Hashim, A. Hamid, and M. Khan, "Multiple solutions for MHD transient flow of Williamson nanofluids with convective heat transport," Journal of the Taiwan Institute of Chemical Engineers, vol. 103, pp. 126-137, 2019.

[5] A. Hamid, M. A. Hashim, M. Khan, and A. S. Alshomrani, "An investigation of thermal and solutal stratification effects on mixed convection flow and heat transfer of Williamson nanofluid," Journal of Molecular Liquids, vol. 284, pp. 307-315, 2019.

[6] S. U. S. Choi, Enhancing Thermal Conductivity of Fluids with Nanoparticles, Int. Mech. Eng. Cong. Exp. ASME, 1995.

[7] S. A. Sajadifar, A. Karimipour, and D. Toghraieb, "Fluid flow and heat transfer of non-Newtonian nanofluid in a microtube considering slip velocity and temperature jump boundary conditions," European Journal of Mechanics - B/Fluids, vol. 61, pp. 25-32, 2017.

[8] W. A. Khan and I. Pop, "Boundary-layer flow of a nanofluid past a stretching sheet," International Journal of Heat and Mass Transfer, vol. 53, no. 11-12, pp. 2477-2483, 2010.

[9] M. Farooq, M. I. Khan, M. Waqas, T. Hayat, A. Alsaedi, and M. I. Khand, "MHD stagnation point flow of viscoelastic nanofluid with non-linear radiation effects," Journal of Molecular Liquids, vol. 221, pp. 1097-1103, 2016.

[10] S. E. Ahmed, R. A. Mohamed, A. M. Aly, and M. S. Soliman, "Magnetohydrodynamic Maxwell nanofluids flow over a stretching surface through a porous medium: effects of non-linear thermal radiation, convective boundary conditions and heat generation/absorption," World Academy of Science, Engineering and Technology International Journal of Aerospace and Mechanical Engineering, vol. 13, pp. 436443, 2019.

[11] A. S. Alshomrani, "On generalized Fourier's and Fick's laws in bio-convection flow of magnetized Burgers nanofluid utilizing motile microorganisms," Mathematics, vol. 8, no. 7, p. 1186, 2020.

[12] A. S. Alshomrani, "Numerical investigation for bio-convection flow of viscoelastic nanofluid with magnetic dipole and motile microorganisms," Arabian Journal for Science and Engineering, vol. 46, no. 6, pp. 5945-5956, 2021.

[13] K. Loganathan and S. Rajan, "An entropy approach of Williamson nanofluid flow with Joule heating and zero nanoparticle mass flux," Journal of Thermal Analysis and Calorimetry, vol. 141, no. 6, pp. 2599-2612, 2020.

[14] W. A. Khan, M. Waqas, W. Chammam, Z. Asghar, U. A. Nisar, and S. Z. Abbas, "Evaluating the characteristics of magnetic dipole for shear-thinning Williamson nanofluid with thermal radiation," Computer Methods and Programs in Biomedicine, vol. 191, article 105396, 2020. 
[15] M. Khan, T. Salahuddin, M. Y. Malik, and F. O. Mallawi, "Change in viscosity of Williamson nanofluid flow due to thermal and solutal stratification," International Journal of Heat and Mass Transfer, vol. 126, pp. 941-948, 2018.

[16] T. Hayat, M. Z. Kiyani, A. Alsaedi, M. I. Khan, and I. Ahmad, "Mixed convective three-dimensional flow of Williamson nanofluid subject to chemical reaction," International Journal of Heat and Mass Transfer, vol. 127, pp. 422-429, 2018.

[17] M. Ramzan, H. Gul, and M. Zahri, "Darcy-Forchheimer 3D Williamson nanofluid flow with generalized Fourier and Fick's laws in a stratified medium," Bulletin of the Polish Academy of Sciences, Technical Sciences, vol. 68, pp. 327-335, 2020.

[18] H. Alfvén, "Existence of electromagnetic-hydrodynamic waves," Nature, vol. 150, no. 3805, pp. 405-406, 1942.

[19] R. A. Mohamed, A. M. Aly, S. E. Ahmed, and M. S. Soliman, "MHD Jeffrey nano fluids flow over a stretching sheet through a porous medium in presence of nonlinear thermal radiation and heat generation/absorption," Transport Phenomena in Nano and Micro Scales, vol. 8, pp. 9-22, 2020.

[20] P. Chandrashekar, B. Nkonga, and A. Bhole, "A discontinuous Galerkin method for a two dimensional reduced resistive MHD model," Computers \& Fluids, vol. 190, pp. 178-191, 2019.

[21] Z.-H. Liu, M.-J. Ni, and N.-M. Zhang, "Numerical study of MHD mixed convection under volumetric heat source in vertical square duct with wall effects," Theoretical and Applied Mechanics Letters, vol. 9, no. 3, pp. 152-160, 2019.

[22] A. Tassone, G. Caruso, F. Giannetti, and A. D. Nevo, "MHD mixed convection flow in the WCLL: heat transfer analysis and cooling system optimization," Fusion Engineering and Design, vol. 146, pp. 809-813, 2019.

[23] S. S. Motsa, P. G. Dlamini, and M. Khumalo, "Spectral relaxation method and spectral quasilinearization method for solving unsteady boundary layer flow problems," Advances in Mathematical Physics, vol. 2014, Article ID 341964, 12 pages, 2014.

[24] A. A. Khan, R. Ellahi, and K. Vafai, "Peristaltic transport of a Jeffrey fluid with variable viscosity through a porous medium in an asymmetric channel," Advances in Mathematical Physics, vol. 2012, Article ID 169642, 15 pages, 2012.

[25] R. Ellahi, "A study on the convergence of series solution of non-Newtonian third grade fluid with variable viscosity by means of homotopy analysis method," Advances in Mathematical Physics, vol. 2012, Article ID 634925, 11 pages, 2012.

[26] M. Umar, R. Akhtar, Z. Sabir et al., "Numerical treatment for the three-dimensional Eyring-Powell fluid flow over a stretching sheet with velocity slip and activation energy," Advances in Mathematical Physics, vol. 2019, Article ID 9860471, 12 pages, 2019.

[27] G. K. Ramesh, B. J. Gireesha, and C. S. Bagewadi, "Heat transfer in MHD dusty boundary layer flow over an inclined stretching sheet with non-uniform heat source/sink," Advances in Mathematical Physics, vol. 2012, Article ID 657805, 13 pages, 2012.

[28] M. Ramzan, M. Farooq, T. Hayat, and J. D. Chunge, "Radiative and Joule heating effects in the MHD flow of a micropolar fluid with partial slip and convective boundary condition," Journal of Molecular Liquids, vol. 221, pp. 394-400, 2016.

[29] R. A. Mohamed, S. M. Abo-Dahab, and T. A. Nofal, “Thermal radiation and MHD effects on free convective flow of a polar fluid through a porous medium in the presence of internal heat generation and chemical reaction," Mathematical Problems in Engineering, vol. 2010, pp. 1-27, 2010.

[30] A. Tetbirt, M. N. Bouaziz, and M. T. Abbes, "Numerical study of magnetic effect on the velocity distribution field in a macro/micro-scale of a micropolar and viscous fluid in vertical channel," Journal of Molecular Liquids, vol. 216, pp. 103-110, 2016.

[31] S. S. Ghadikolaei, K. Hosseinzadeh, and D. D. Ganjib, "Numerical study on magnetohydrodynic CNTs-water nanofluids as a micropolar dusty fluid influenced by non-linear thermal radiation and Joule heating effect," Powder Technology, vol. 340, pp. 389-399, 2018.

[32] J. Gireesha, B. Mahanthesh, G. T. Thammanna, and P. B. Sampathkumar, "Hall effects on dusty nanofluid two-phase transient flow past a stretching sheet using KVL model," Journal of Molecular Liquids, vol. 256, pp. 139-147, 2018.

[33] A. Aghanajafi, D. Toghraie, and B. Mehmandoust, "Numerical simulation of laminar forced convection of water- $\mathrm{CuO}$ nanofluid inside a triangular duct," Physica E: Low-dimensional Systems and Nanostructures, vol. 85, pp. 103-108, 2017.

[34] A. Hussain, M. Y. Malik, T. Salahuddin, S. Bilal, and M. Awais, "Combined effects of viscous dissipation and Joule heating on MHD Sisko nanofluid over a stretching cylinder," Journal of Molecular Liquids, vol. 231, pp. 341-352, 2017.

[35] C. S. K. Raju, N. Sandeep, and A. Malvandi, "Free convective heat and mass transfer of MHD non-Newtonian nanofluids over a cone in the presence of non-uniform heat source/sink," Journal of Molecular Liquids, vol. 221, pp. 108-115, 2016.

[36] T. Hayat, S. A. Shehzad, M. Qasim, and S. Obaidat, "Radiative flow of Jeffery fluid in a porous medium with power law heat flux and heat source," Nuclear Engineering and Design, vol. 243, pp. 15-19, 2012.

[37] J. P. Hartnett and M. Kostic, "Heat transfer to Newtonian and non-Newtonian fluids in rectangular ducts," Advances in Heat Transfer, vol. 19, pp. 247-356, 1989.

[38] I. Dapra and G. Scarpi, "Perturbation solution for pulsatile flow of a non-Newtonian Williamson fluid in a rock fracture," International Journal of Rock Mechanics and Mining Sciences, vol. 44, no. 2, pp. 271-278, 2007.

[39] S. Nadeem and S. T. Hussain, "Flow and heat transfer analysis of Williamson nanofluid," Applied Nanoscience, vol. 4, no. 8, pp. 1005-1012, 2014. 